\title{
Numerical Modelling of Interaction between Hydraulic Fractures and Natural Fractures by Using the Extended Finite Element Method
}

\author{
Peilun Li, ${ }^{1}$ Yan Dong, ${ }^{2}$ Sheng Wang, ${ }^{1}$ and Peichao Li $\mathbb{D}^{3}$ \\ ${ }^{1}$ Reservoir Dynamic Monitoring Center, Shengli Oilfield Company, SINOPEC, Dongying 257015, China \\ ${ }^{2}$ Department of Modern Mechanics, University of Science and Technology of China, Hefei 230027, China \\ ${ }^{3}$ School of Mechanical and Automotive Engineering, Shanghai University of Engineering Science, Shanghai 201620, China \\ Correspondence should be addressed to Peichao Li; wiselee18@163.com
}

Received 17 June 2020; Revised 1 August 2020; Accepted 5 September 2020; Published 21 September 2020

Academic Editor: Richeng Liu

Copyright (c) 2020 Peilun Li et al. This is an open access article distributed under the Creative Commons Attribution License, which permits unrestricted use, distribution, and reproduction in any medium, provided the original work is properly cited.

Natural fractures usually develop in shale reservoirs. Thereby, in the process of hydraulic fracturing, it is inevitable that hydraulic fractures will intersect with natural fractures. In order to reveal the interaction mechanism between hydraulic-induced fractures and natural fractures, a two-dimensional fracture intersection model based on the extended finite element method (XFEM) is proposed, and the different types of intersecting criteria reported in the literature are compared. Then, the effects of natural fracture azimuth, fluid pressure in hydraulic fracture, and in situ principal stress difference on hydraulic fracturing are studied in detail. The results show that the fracture morphology is different under different criteria and working conditions. And the stress concentration phenomenon mainly concentrates on the tip in the obtuse angle side of natural fracture. Meanwhile, different fluid pressures in hydraulic fracture can also induce different intersection patterns. The obtained results in this work are of great benefit to understand the intersection mechanism between hydraulic fractures and natural fractures.

\section{Introduction}

Generally, shale reservoirs have very low porosity and permeability. Thus, hydraulic fracturing technique is widely employed to generate hydraulic/induced fractures and enhance the productivity of shale reservoirs. There are many discontinuities in shale reservoirs, such as natural fractures/cracks, bedding planes, and faults [1]. Natural fracture is probably one major reason for possible change in hydraulic fracture morphology from what it was originally expected. In other words, the interaction between hydraulic fractures and natural fractures arises commonly in naturally fractured low-permeability reservoirs [2]. Meanwhile, natural fractures have production capacity only when they are activated by induced fractures [3]. Thus, understanding the interaction mechanism between hydraulic fractures and natural fractures is of great significance for the stimulation of shale reservoirs.
Extensive theoretical works have been conducted to investigate the interaction mechanism between hydraulic fractures and natural fractures based on experiments [2, 4-6]. Gu and Weng [7] extended the Renshaw and Pollard results to apply to the nonorthogonal intersecting angle condition. Compared to theoretical and experimental methods, numerical methods can simulate the interaction process under more complex geological conditions. Numerous numerical works have been carried out on the basis of the discrete element method (DEM) [8] and the displacement discontinuity method (DDM) [9-11]. Zhang et al. [12] investigated the interaction mechanism between hydraulic fractures and natural fractures with a hybrid discrete-continuum method. Huang et al. [13] proposed different 3D perforation models of hydraulic fracture initiation and near-wellbore propagation. And, the pressure characteristics and performance of multistage fractured horizontal well have been analyzed with coupled flow and 
geomechanics [14]. In addition, the full coupled multiscale numerical model has been introduced to investigate hydraulic fracturing complexity in naturally fractured rock masses [15]. Belytschko and Black [16] presented a minimal remeshing finite element method for crack growth, which is referred to as the extended finite element method (XFEM). Therein, the enrichment functions are added to the finite element approximation to account for the presence of the cracks. The XFEM allows the crack to be arbitrarily aligned within the mesh, overcomes the limitations inherent in the mesh shape, and can efficiently simulate fractures without remeshing. Dahi-Taleghani and Olson $[17,18]$ investigated the intersection between hydraulic fractures and natural fractures based on a plane strain XFEM model. Xu et al. [19] developed the additional junction enrichment to describe branched elements. Wang et al. [20] established a fluid-solid coupled hydraulic fracturing model using the XFEM to simulate the interactions between hydraulic fractures and natural fractures and further investigate the formation of fracture network.

There are a lot of criteria for the intersection. It is important to adopt the most appropriate criterion in different situations. That is, the intersection between hydraulic fractures and natural fractures still needs further study. Therefore, a two-dimensional crack intersection model based on the XFEM is proposed in this work. In addition, different types of intersection criteria reported in previous studies are used to numerically simulate the interaction in the fracturing process.

\section{Intersection Model}

The intersection of hydraulic fractures and natural fractures is a complex problem involving rock deformation, fluid flow and distribution at the joint point, and influence of in situ stress field. In general, there are four typical patterns of interaction as follows [21]:

(1) When fluid pressure in hydraulic fracture is small, the induced fracture will be arrested due to the energy dissipation of induced fracture.

(2) If fluid pressure of hydraulic fracture is large enough to open the natural fracture, the hydraulic fracture will deflect to the natural fracture and propagate along the direction of natural fracture.

(3) If the natural fracture is very difficult to open, while fluid pressure in hydraulic fracture is large enough, the natural fracture will be penetrated, and the hydraulic fracture will propagate along the initial pathway.

(4) If the natural fracture is easy to open and fluid pressure in hydraulic fracture is large enough, the hydraulic fracture may penetrate the natural fracture while the natural fracture is opened simultaneously.

In this section, we present a brief review of several analytical criteria of interaction between hydraulic fractures and natural fractures and explain how they can be applied in the XFEM.

2.1. Blanton's Criterion. Blanton's criterion [4] considers that when hydraulic fracture reaches natural fracture plane, the fluid pressure at the intersection point will continue to rise until the induced fracture either opens or penetrates the natural fracture. If the required pressure for reinitiation is lower than the opening pressure, the induced fracture will penetrate the natural fracture and continue to propagate along the original direction. As shown in Figure 1, fluid pressure $p$ is imposed on the hydraulic fracture plane, $\theta$ is the angle of approach, $\sigma_{\mathrm{hmax}}$ and $\sigma_{\mathrm{hmin}}$ are the maximum and minimum in situ horizontal principal stresses, and $\sigma_{n}$ and $\tau$ are the normal and shear stresses acting on the natural fracture plane, respectively. Also, $\sigma_{T}$ is the normal stress component acting parallel to the natural fracture which depends on the in situ stresses, fluid pressure, and geometry of the interaction region as well as friction coefficient. The crossing criterion is described as follows:

$$
p>\sigma_{T}+T_{0},
$$

where $T_{0}$ is the tensile strength of the rock. Accordingly, Blanton derived the following crossing criterion:

$$
\frac{\sigma_{\mathrm{h} \text { max }}-\sigma_{\mathrm{hmin}}}{T_{0}}>\frac{-1}{\cos 2 \theta-b \sin 2 \theta},
$$

where $\sigma_{\mathrm{hmax}}-\sigma_{\mathrm{hmin}}$ is called the in situ horizontal principal stress difference.

Here, $b$ is defined as

$$
b=\frac{1}{2 c}\left[v\left(x_{0}\right)-\frac{\left(x_{0}-l\right)}{\mu_{f}}\right],
$$

where $x_{0}$ is the point at which reinitiation will occur, $c$ is the zone of slippage in the natural fracture, $l$ is the half length of crack, and $\mu_{f}$ is the friction coefficient of the natural fracture plane. $x_{0}$ and $v\left(x_{0}\right)$ are given by

$$
\begin{aligned}
x_{0} & =\left[\frac{(1+c)^{2}+e^{\left(\pi /\left(2 \mu_{f}\right)\right)}}{l+e^{\left(\pi /\left(2 \mu_{f}\right)\right.}}\right]^{0.5}, \\
v\left(x_{0}\right) & =\frac{1}{\pi}\left[\left(x_{0}+l\right) \ln \left(\frac{x_{0}+l+c}{x_{0}+l}\right)^{2}+\left(x_{0}-l\right) \ln \left(\frac{x_{0}-l-c}{x_{0}-l}\right)^{2}+c \ln \left(\frac{x_{0}+l+c}{x_{0}-l-c}\right)^{2}\right] .
\end{aligned}
$$




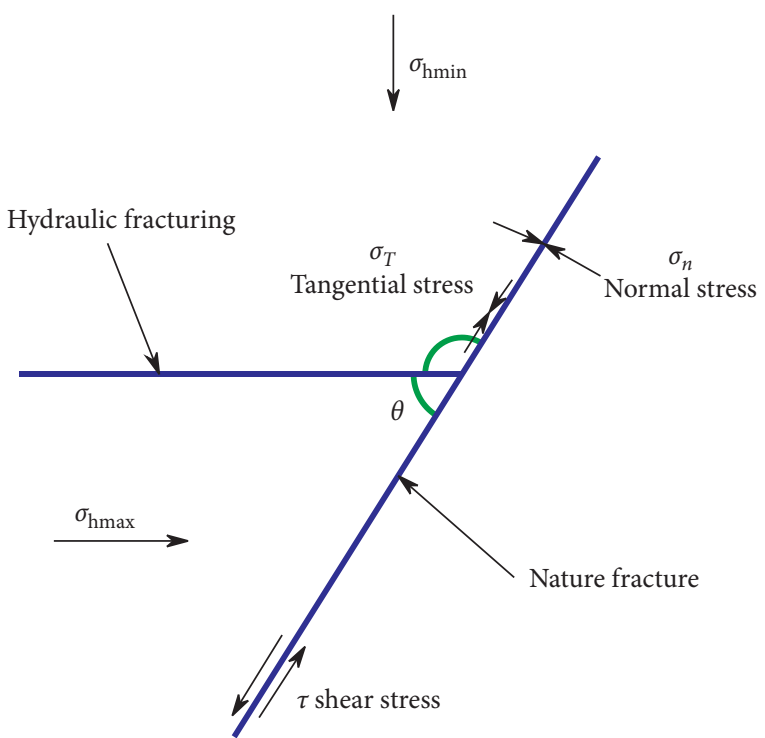

FIGURE 1: Schematic of a hydraulic fracture intersecting a natural fracture.

When the tensile strength of rock is $3.5 \mathrm{MPa}$, the chart of Blanton's criterion as shown in Figure 2 can be obtained. In addition, Blanton's criterion ignores the influence of fluid pressure on the induced stress at the natural fracture interface and assumes that the shear stress is a simple linear distribution along the natural fracture interface.

2.2. WङT's Criterion. Warpinski and Teufel [5] claim that the forming of intersection is determined by the net fluid pressure, $p_{n}$, which is defined as the fluid pressure in hydraulic fracture minus the minimum horizontal stress. Opening will take place if the induced pressure exceeds the far-field stress component acting normal to the natural fracture plane. The opening condition can be expressed as

$$
\sigma_{\text {hmax }}-\sigma_{\text {hmin }}<\frac{2 p_{n}}{1-\cos 2 \theta} .
$$

Beyond that, Warpinski and Teufel proposed a relationship expression that governs arrest mode as

$$
\sigma_{\text {hmax }}-\sigma_{\text {hmin }} \geq \frac{2 \tau_{0}-2 p_{n} \mu_{f}}{\sin 2 \theta+\mu_{f} \cos 2 \theta-\mu_{f}},
$$

where $\tau_{0}$ is the shear strength.

The chart of W\&T's criterion has been plotted in Figures 3 and 4 corresponding to net fluid pressures of $0.7 \mathrm{MPa}$ and $2.7 \mathrm{MPa}$, respectively.

It can be seen that there is a critical angle, denoted by $\alpha$, in the W\&T's criterion plate curve. When $\theta>\alpha$, shear slippage will not take place because normal stress on the natural fracture plane is big enough to stop the slippage. At that time, the hydraulic crack would either penetrate the natural fracture or be arrested. The critical angle can be derived from (6), which is given by

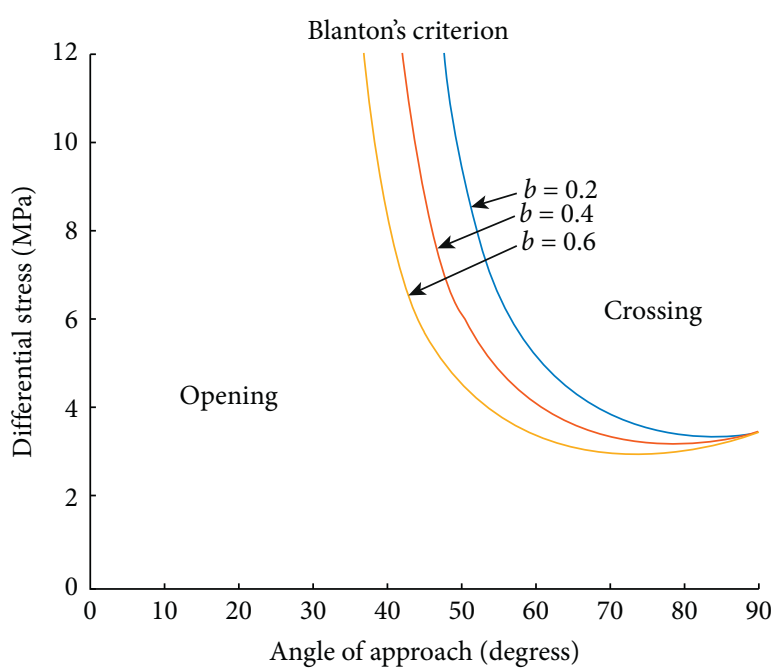

Figure 2: The chart of Blanton's criterion.

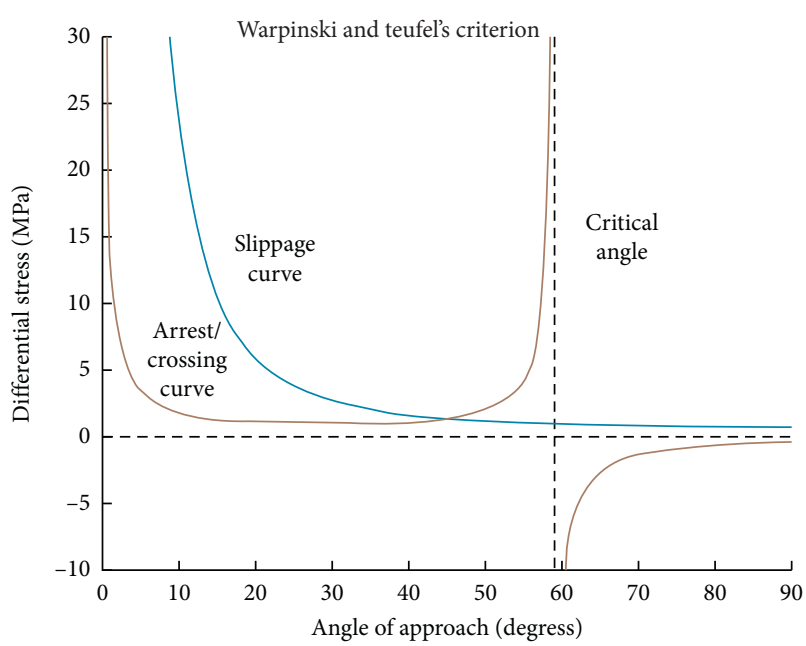

Figure 3: The chart of W\&T's criterion $\left(p_{n}=0.7 \mathrm{MPa}\right)$.

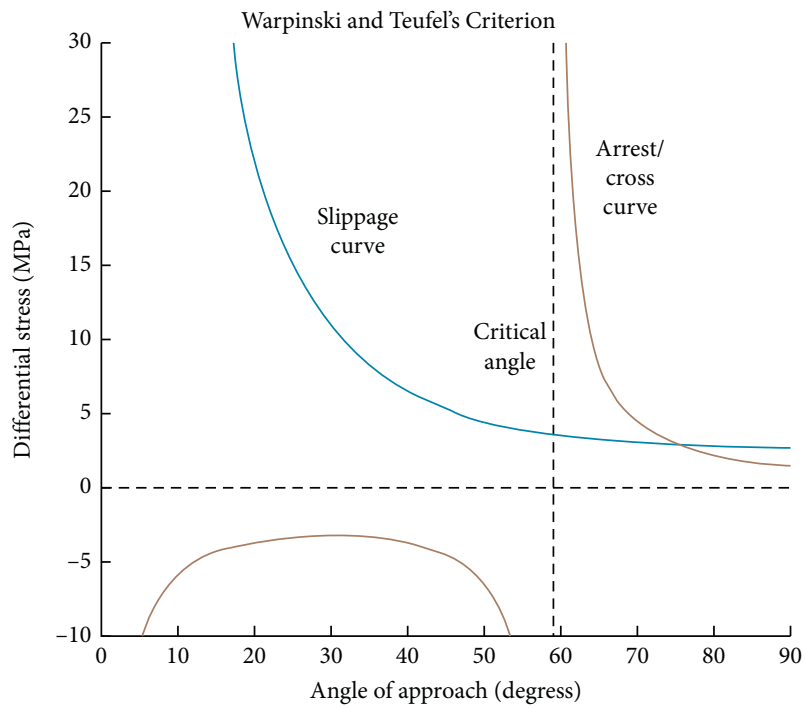

Figure 4: The chart of W\&T's criterion $\left(p_{n}=2.7 \mathrm{MPa}\right)$. 


$$
\alpha=\arctan \left(\frac{1}{\mu_{f}}\right)
$$

2.3. R\&P's Criterion. With considering that the material is homogeneous and isotropic, Renshaw and Pollard [6] studied the interaction of a dry fracture with a frictional plane based on the elastic fracture theory. This criterion considers the case where induced fracture approaching natural fracture orthogonally. If the shear strength of the natural fracture is less than the induced tensile stress, the natural fracture will slip and the fracture will be arrested. The criterion can be expressed as

$$
\frac{-\sigma_{\mathrm{hmax}}}{T_{0}-\sigma_{\mathrm{hmin}}}>\frac{1+\mu_{f}}{\mu_{f}}
$$

Renshaw and Pollard propose that crossing will not take place if slippage occurs, which underestimates the discontinuity strength required to allow an induced fracture to cross it. The chart of R\&P's criterion is shown in Figure 5.

2.4. Modified R\&P's Criterion. In fact, the induced crack may approach the natural fracture with any angle, so it is necessary to improve the R\&P's intersection criterion and

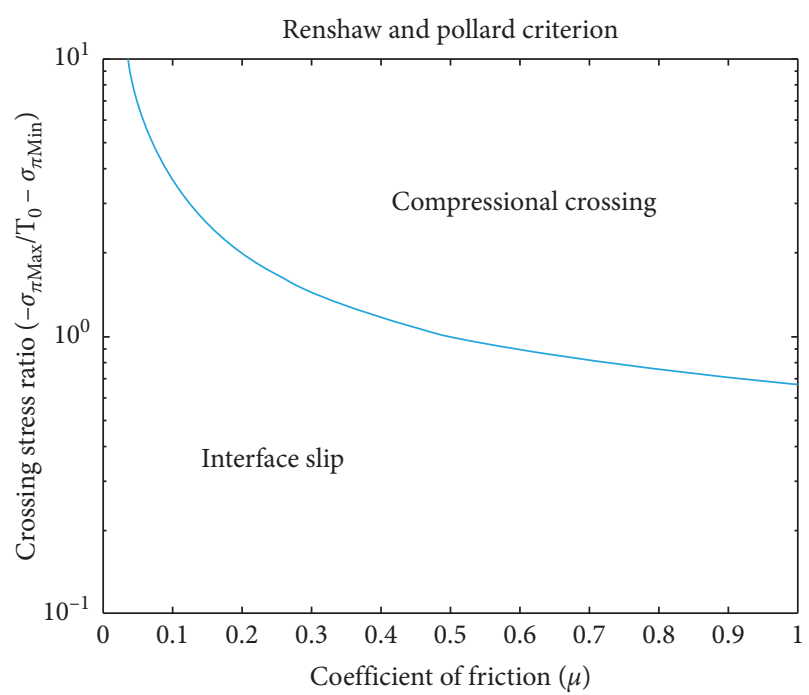

FIgURE 5: The curve of R\&P's criterion.

develop a more general applicability one. Sarmadivaleh and Rasouli [2] extended R\&P's criterion to the case of a noncohesive interface with a nonorthogonal approach angle. It can be expressed as

$$
\frac{-\sigma_{n}}{\left(T_{0}-\sigma_{T}\right)}>\frac{(1-\sin (\theta / 2) \sin (3 \theta / 2))+\left(1 / \mu_{f}^{\prime \prime} \cos (\theta / 2)\right)(|\sin (\theta / 2) \cos (\theta / 2) \cos (3 \theta / 2)|+\alpha)}{(1+\sin (\theta / 2) \sin (3 \theta / 2))},
$$

where $\mu_{f}^{\prime \prime}=\mu_{f}^{\prime}+\mu_{f}$ and is called the total friction coefficient. Here, $\mu_{f}^{\prime}$ is the relative friction coefficient, denoted as follows:

$$
\mu_{f}^{\prime}=\frac{\tau_{0} / \sigma_{n}}{\left(((1-\sin (\theta / 2) \sin (3 \theta / 2))) /\left((1-\sin (\theta / 2) \sin (3 \theta / 2))+\left(1 / \mu_{f} \cos (\theta / 2)\right)(|\sin (\theta / 2) \cos (\theta / 2) \cos (3 \theta / 2)|+\alpha)\right)\right)-1}
$$

\section{Numerical Method}

Here, we employ the XFEM [16] to simulate the studied problem. The element with no crack is regarded as normal element, which is the same as the standard FEM. Note that for crack-crossed elements, the Heaviside step function is introduced as enriched function to characterize the discontinuities of crack. That is,

$$
H(x)= \begin{cases}1, & \operatorname{dist}(x)>0 \\ -1, & \operatorname{dist}(x)<0\end{cases}
$$

where $\operatorname{dist}(x)$ is the shortest distance from $x$ to crack. In order to determine whether it is positive or negative, $n$ is defined as the unit normal vector to the crack. The distance is positive when $x$ locates in the side where $n$ points and it is negative when $x$ locates in the other side [21].

For embedded elements, Westergaad function is introduced as enriched function, which can be expressed as

$$
\phi_{\alpha}(r, \theta)=\sqrt{r}\left[\sin \frac{\theta}{2}, \cos \frac{\theta}{2}, \sin \frac{\theta}{2} \sin \theta, \cos \frac{\theta}{2} \sin \theta\right],
$$

where $r$ and $\theta$ are the local parameters in crack-tip polar coordinates: 


$$
\left\{\begin{array}{l}
r=\sqrt{\left(x-x_{\text {tip }}\right)^{2}+\left(y-y_{\text {tip }}\right)^{2}} \\
\theta=\arctan \frac{y-y_{\text {tip }}}{x-x_{\text {tip }}}-\theta_{\text {tip }}
\end{array}\right.
$$

where $\theta_{\text {tip }}$ is the crack-tip angle in the orthogonal coordinate system.

In the framework of XFEM, the node displacement approximation is [9]

$$
\mathbf{u}^{h}(x)=\sum_{i \in \mathbf{N}} N_{i}(x)[\mathbf{u}_{i}+\underbrace{H(x) \mathbf{a}_{i}}_{i \in \mathbf{N}_{H}}+\underbrace{\sum_{\alpha=1}^{4} \phi_{\alpha} \mathbf{b}_{i}^{\alpha}}_{i \in \mathbf{N}_{\mathrm{emb}}}],
$$

where $\mathbf{N}$ is all nodes set, $\mathbf{N}_{H}$ is the set of nodes in crackcrossed elements, and $\mathbf{N}_{e m b}$ is the set of nodes in embedded elements. $N_{i}(x)$ is the classical shape function, $\mathbf{u}_{i}$ is the continuous degrees-of-freedom (DOFs) vector, and $\mathbf{a}_{i}$ and $\mathbf{b}_{i}^{\alpha}$ are the enriched DOFs vector of crack-crossed elements and embedded elements, respectively.

The enrichment of the element crossed by two separate cracks is given as follows [20]:

$$
u^{h}(X)=\sum_{I=1}^{4} N_{I}(X) u_{I}+\sum_{J=1}^{4} N_{J}(X)\left[H\left(f^{I}(X)\right)-H\left(f^{I}\left(X_{J}\right)\right)\right] q_{J}^{I}+\sum_{K=1}^{4} N_{K}(X)\left[H\left(f^{I I}(X)\right)-H\left(f^{I I}\left(X_{K}\right)\right)\right] q_{K}^{I I}
$$

where $f^{\mathrm{I}}(\mathbf{X})$ and $f^{\mathrm{I}}(\mathbf{X})$ are the signed distance functions. Also, $\mathbf{q}_{\mathrm{J}}^{\mathrm{I}}$ and $\mathbf{q}^{\mathrm{II}}{ }_{\mathrm{k}}$ are the enriched DOFs of main crack and the branch crack, respectively.
The enrichment of the element containing a junction is as follows:

$$
u^{h}(X)=\sum_{I=1}^{4} N_{I}(X) u_{I}+\sum_{J=1}^{4} N_{J}(X)\left[H\left(f^{I}(X)\right)-H\left(f^{I}\left(X_{J}\right)\right)\right] q_{J}^{I}+\sum_{K=1}^{4} N_{K}(X)\left[J(X)-J\left(X_{K}\right)\right] q_{K}^{I I}
$$

Here, a junction function is introduced as

$J(\mathbf{X})= \begin{cases}H\left(f^{\mathrm{I}}(\mathbf{X})\right), & \text { on the side without branch, } \\ H\left(f^{\mathrm{II}}(\mathbf{X})\right), & \text { on the side with branch. }\end{cases}$
The enrichment of the element containing an intersection is defined as follows:

$$
u^{h}(X)=\sum_{I=1}^{4} N_{I}(X) u_{I}+\sum_{J=1}^{4} N_{J}(X)\left[H\left(f^{I}(X)\right)-H\left(f^{I}\left(X_{J}\right)\right)\right] q_{J}^{I}+\sum_{K=1}^{4} N_{K}(X)\left[J^{1}(X)-J^{1}\left(X_{K}\right)\right] c_{K}^{1}+\sum_{K=1}^{4} N_{K}(X)\left[J^{2}(X)-J^{2}\left(X_{K}\right)\right] c_{K}^{2},
$$

where $q_{J}^{I}, c_{K}^{1}$, and $c_{K}^{2}$ are the enriched DOFs of main crack and two branch cracks, respectively.

\section{Results and Discussion}

4.1. Effect of Natural Fracture Azimuth. In this section, we study the influence of natural fracture azimuth on the hydraulic fracture propagation. The model is $100 \mathrm{~m} \times 100 \mathrm{~m} \times 1 \mathrm{~m}$ in size. A linear elastic and isotropic block is considered with Young's modulus of $25 \mathrm{GPa}$ and Poisson's ratio of 0.20 , and the tensile strength $T_{0}$ is $1.0 \mathrm{MPa}$. The in situ principal stress difference is set as $2.0 \mathrm{MPa}$, and fluid pressure in hydraulic fracture is $10 \mathrm{MPa}$. With seeding
101 nodes each side, the model is divided into 10000 elements. The initial induced crack, with a length of $5 \mathrm{~m}$, is set at the left boundary. Three cases of different natural fractures orientations are studied. As shown in Figure 6, natural fractures with horizontal angles of $30^{\circ}, 45^{\circ}$, and $60^{\circ}$ are set in the block.

The results based on Blanton's criterion are illustrated in Figure 7. In this case, hydraulic fracture opens one side of the natural fracture and reinitiate in the opposite plane. That is, the offset of hydraulic fracture occurs in the $y$ direction. And the induced fracture's offset will be larger if the natural fracture azimuth is bigger. When the in situ principal stress difference is $2.0 \mathrm{MPa}$, the induced crack is 


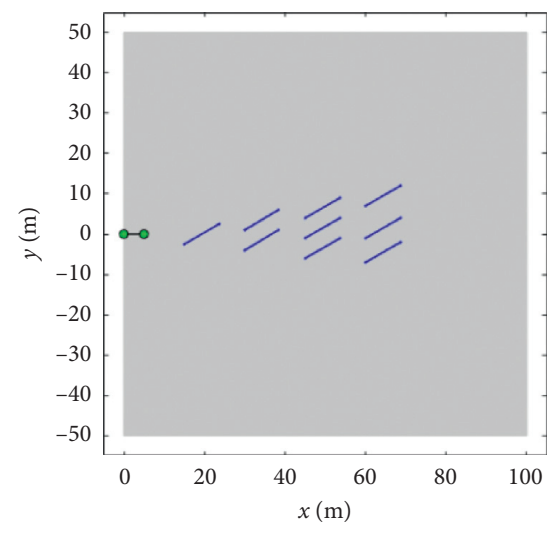

(a)

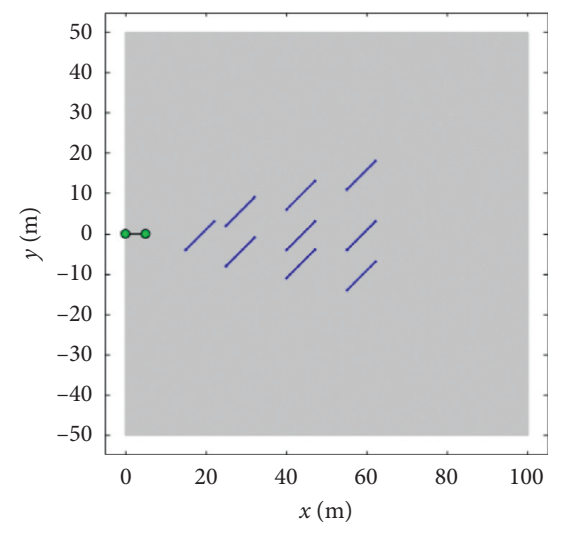

(b)

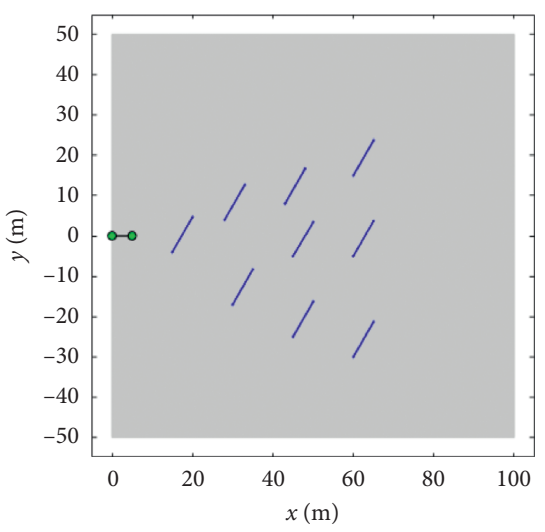

(c)

FiguRE 6: Initial fracture distribution. (a) Initial crack distribution, $30^{\circ}$. (b) Initial crack distribution, $45^{\circ}$. (c) Initial crack distribution, $60^{\circ}$.

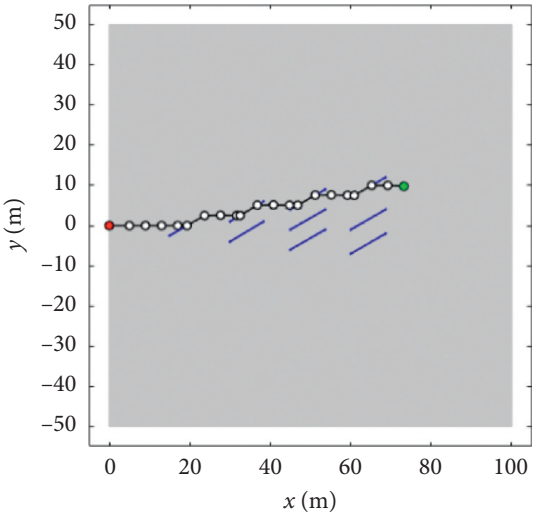

(a)

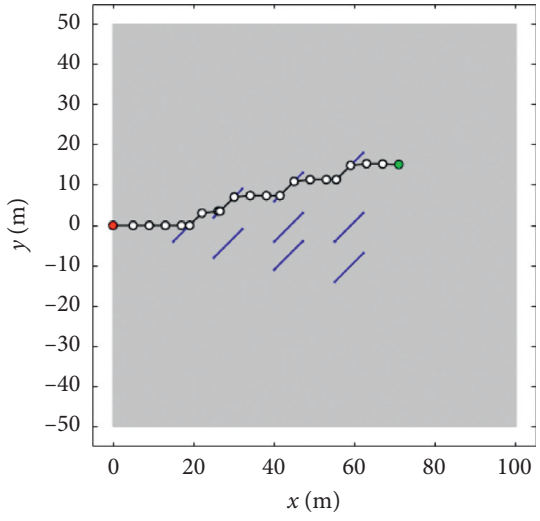

(b)

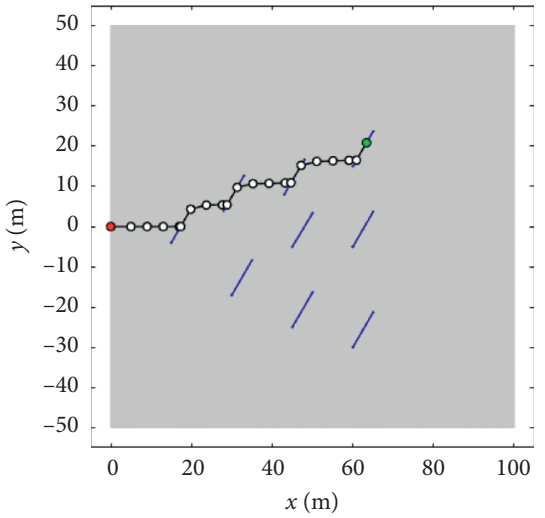

(c)

Figure 7: Fracture propagation morphology under Blanton's criterion. (a) Final fracture, $30^{\circ}$. (b) Final fracture, $45^{\circ}$. (c) Final fracture, $60^{\circ}$.

impossible to penetrate the natural fracture regardless of the crack angle. The propagation behavior of hydraulic fracture in this work agrees well with that of previous study [9].

As we can see from Figure 8, when the angle of intersection is $30^{\circ}$, the stress concentration phenomenon mainly concentrates on the upper tip of the natural fracture. And the induced stress at the lower tip of the natural fracture is smaller as it is suppressed by the fluid pressure. The impact of squeezing will be reduced when the intersection angle gradually increases. When the intersection angle reaches $135^{\circ}$, the stress concentration phenomenon mainly occurs at the lower tip of the crack, which is exactly contrary to the $45^{\circ}$ case. In Wang et al.'s study [9], the stress distribution along the natural fracture becomes asymmetrical and the stress concentration is more significant in the vicinity of the upper tip of the natural fracture.

4.2. Effect of In Situ Principal Stress Difference. In order to examine the influence of the in situ principal stress difference on hydraulic fracture propagation, we compare the results of two cases with the in situ principal stress difference of $2 \mathrm{MPa}$ and $4 \mathrm{MPa}$. Natural fractures with horizontal angle of $60^{\circ}$ are set in the block. The shape of induced fracture after the intersection is displayed in Figure 9. Different from before, the offset of hydraulic crack does not occur. Hydraulic fracture penetrates the natural fractures without changing the propagation direction when the in situ principal stress difference is $4 \mathrm{MPa}$. When the principal stress difference increases, hydraulic fracture requires a larger driving fluid pressure to obtain a larger net fluid pressure to open natural fractures. But at this time, the net fluid pressure is enough to induce re initiation occurring on the other side of natural fracture. Therefore, the hydraulic fracture will be inclined to penetrate the natural fracture when the in situ principal stress difference increases to a certain degree.

4.3. Effect of Fluid Pressure. In order to examine the influence of fluid pressure on the hydraulic fracture propagation, the intersection criterion is changed into the W\&T's criterion. Natural fractures with horizontal angle of $30^{\circ}$ are set in the block. And the in situ principal stress difference is set 


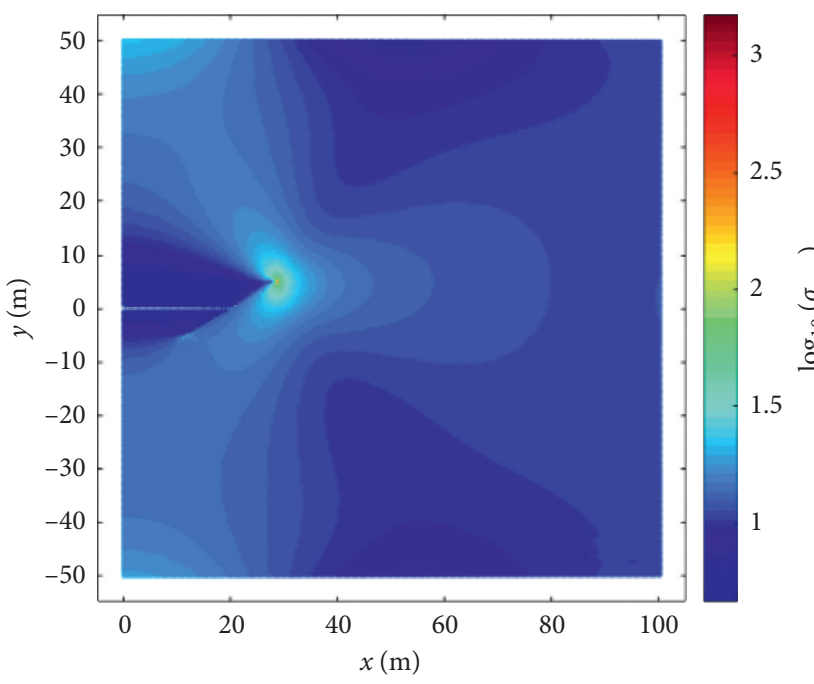

(a)

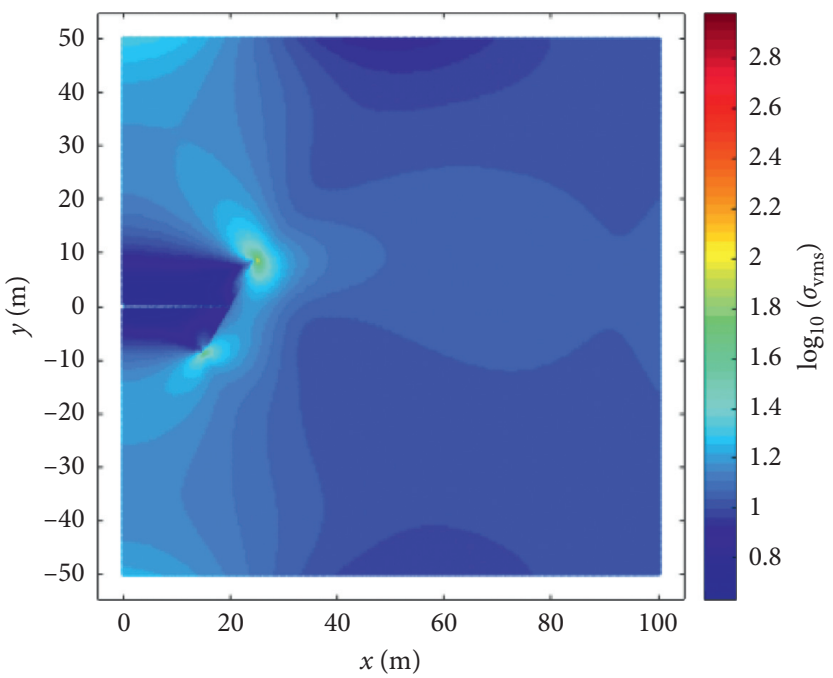

(c)

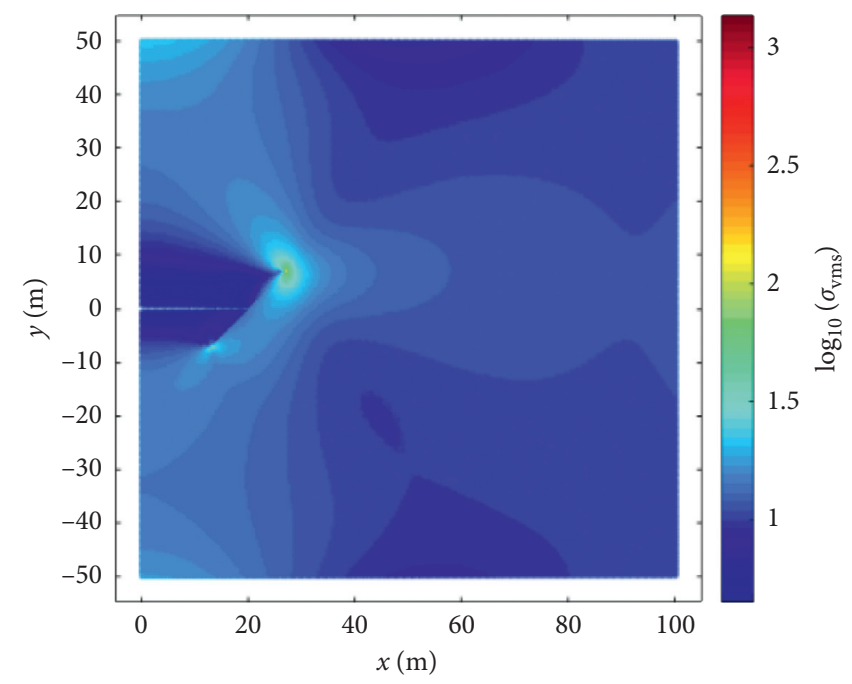

(b)

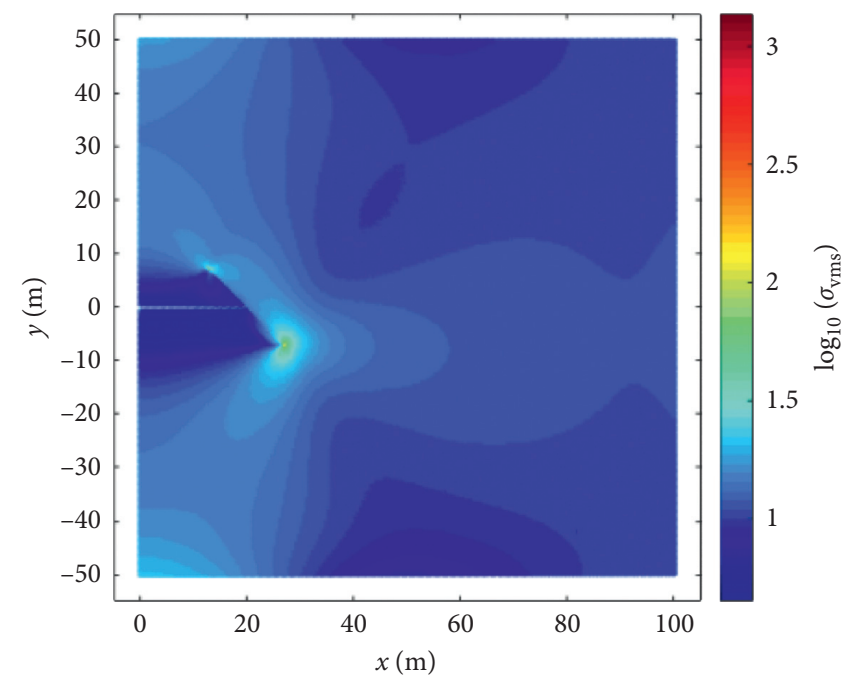

(d)

Figure 8: Von Mises stress field under different natural fracture azimuth conditions. (a) von Mises stress (step $=3$ ), $30^{\circ}$. (b) von Mises stress $($ step $=3), 45^{\circ}$. (c) von Mises stress $($ step $=3), 60^{\circ}$. (d) von Mises stress (

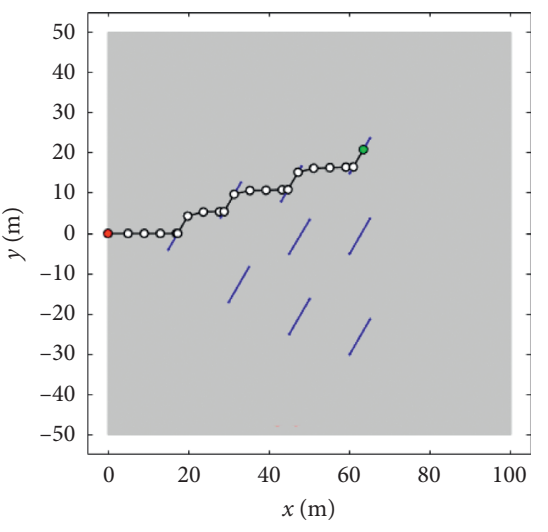

(a)

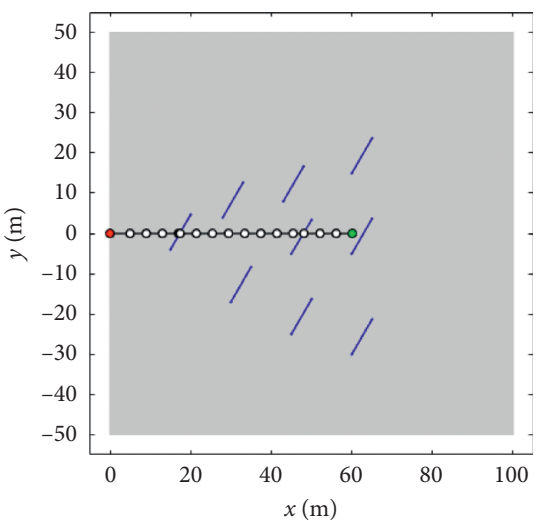

(b)

FIGURE 9: Intersection results based on Blanton's criterion under different in situ principal stress difference conditions. (a) In situ principal stress difference is $2 \mathrm{MPa}$; final fracture, $60^{\circ}$. (b) In situ principal stress difference is $4 \mathrm{MPa}$; final fracture, $60^{\circ}$. 


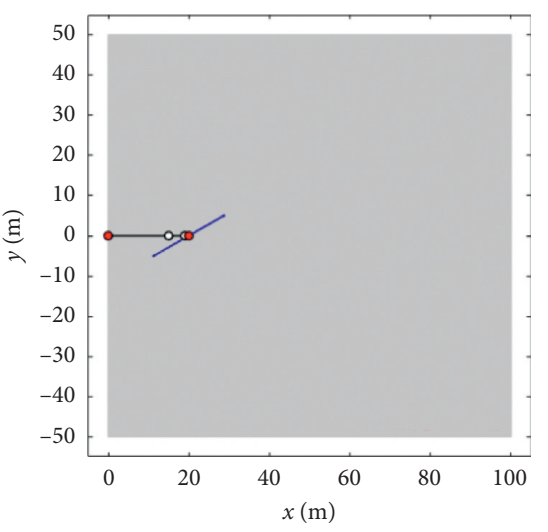

(a)

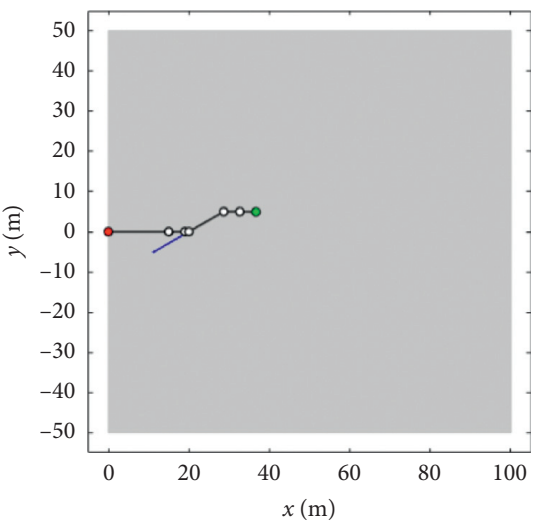

(c)

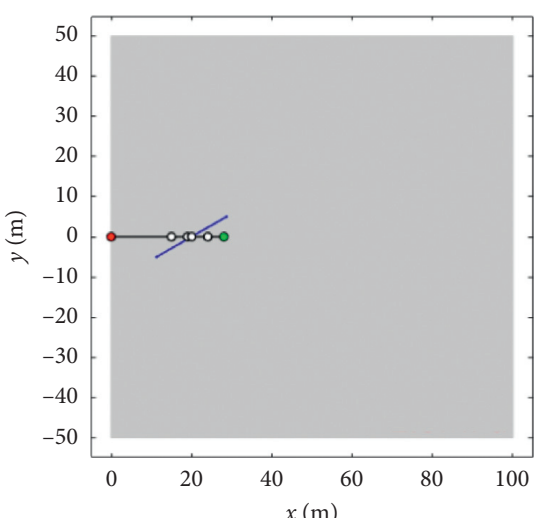

(b)

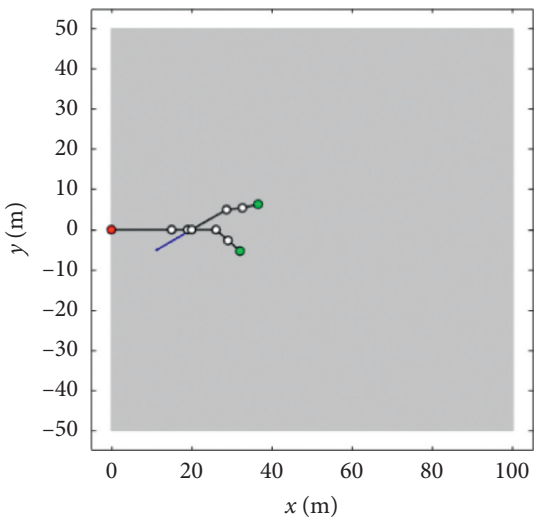

(d)

FIGURE 10: Intersection results based on the W\&T's criterion under different fluid pressure conditions. (a) Final fracture, 2.5 MPa. (b) Final fracture, 2.8 MPa. (c) Final fracture, 3.5 MPa. (d) Final fracture, 4.5 MPa.

as 2.0 MPa. The shapes of induced fracture of different cases are shown in Figure 10. We compare the results of four cases with the fluid pressure of $2.5 \mathrm{MPa}, 2.8 \mathrm{MPa}, 3.5 \mathrm{MPa}$, and 4.5 $\mathrm{MPa}$. The shear strength and the friction coefficient are set as $0.7 \mathrm{MPa}$ and $0.6 \mathrm{MPa}$.

It can be found from Figure 10 that there are four intersection modes after the intersection of hydraulic crack and natural fracture. When the fluid pressure is $2.5 \mathrm{MPa}$, the net fluid pressure is too small to offset the normal stress in the natural fracture surface. At this point, the hydraulic crack will be arrested when it encounters the natural fracture. When the fluid pressure increases to $2.8 \mathrm{MPa}$, the induced stress is big enough to make the hydraulic fracture penetrate the natural fracture. When the pressure continues to increase to $3.5 \mathrm{MPa}$, the condition of opening is satisfied at this time, and the hydraulic crack is deflected into the direction of natural fracture. The induced stress can simultaneously meet the conditions of penetration and opening when the fluid pressure increases to $4.5 \mathrm{MPa}$. Thus, two branch cracks generate at this time.

\section{Conclusions}

In this paper, we compare several intersection criteria and simulate the interaction process between hydraulic fractures and natural fractures based on the XFEM. The effects of natural fracture azimuth, fluid pressure, and in situ principal stress difference on hydraulic fracturing are investigated in detail. The main conclusions are summarized as follows:

(1) Blanton's criterion is applicable to the situation where the natural fracture interface has a high normal stress. Also, the W\&T's criterion considers that the natural fracture friction coefficient and shear strength are also the influencing factors of crack propagation. The modified R\&P's criterion takes into account the cohesive effects of natural fracture.

(2) The stress concentration phenomenon mainly concentrates on the tip in the obtuse angle side of natural fracture. Also, the induced fracture's offset becomes larger with the increase of the natural fracture azimuth.

(3) Hydraulic fracture is inclined to penetrate the natural fracture when the in situ principal stress difference increases to a certain extent.

(4) Different fluid pressures in hydraulic fracture may trigger different intersection patterns, such as penetration mode, arrest mode, offset mode, and simultaneity mode. 


\section{Data Availability}

The data used to support the findings of this work are available from the corresponding author upon request.

\section{Conflicts of Interest}

The authors declare that there are no conflicts of interest regarding the publication of this paper.

\section{Acknowledgments}

This work was funded by the National Science and Technology Major Project of China (Grant no. 2017ZX05072) and the Natural Science Foundation of Shanghai (Grant no. 19ZR1421400).

\section{References}

[1] J. F. W. Gale, R. M. Reed, and J. Holder, "Natural fractures in the Barnett Shale and their importance for hydraulic fracture treatments," AAPG Bulletin, vol. 91, no. 4, pp. 603-622, 2007.

[2] M. Sarmadivaleh and V. Rasouli, "Modified Reinshaw and Pollard criteria for a non-orthogonal cohesive natural interface intersected by an induced fracture," Rock Mechanics and Rock Engineering, vol. 47, no. 6, pp. 2107-2115, 2014.

[3] G. E. King, L. Haile, J. A. Shuss, and T. Dobkins, "Increasing fracture path complexity and controlling downward fracture growth in the Barnett shale," in Proceedings of the SPE 119896 Presented at the Shale Gas Production Conference, Fort Worth, TX, USA, November 2008.

[4] T. L. Blanton, "Propagation of hydraulically and dynamically induced fractures in naturally fractured reservoirs," in Proceedings of the SPE 15261 Presented at Unconventional Gas Technology Symposium, Louisville, KY, USA, May 1986.

[5] N. R. Warpinski and L. W. Teufel, "Influence of geologic discontinuities on hydraulic fracture propagation (includes associated papers 17011 and 17074)," Journal of Petroleum Technology, vol. 39, no. 2, pp. 209-220, 1987.

[6] C. E. Renshaw and D. D. Pollard, "An experimentally verified criterion for propagation across unbounded frictional interfaces in brittle, linear elastic materials," International Journal of Rock Mechanics and Mining Sciences \& Geomechanics Abstracts, vol. 32, no. 3, pp. 237-249, 1995.

[7] H. Gu and X. Weng, "Criterion for fractures crossing frictional interfaces at non-orthogonal angles," in Proceedings of the 44th US Rock Mechanics Symposium and 5th US-Canada Rock Mechanics Symposium, New York, NY, USA, 2010.

[8] Y. Zou, S. Zhang, X. Ma, T. Zhou, and B. Zen, "Numerical investigation of hydraulic fracture network propagation in naturally fractured shale formations," Journal of Structural Geology, vol. 84, pp. 1-13, 2016.

[9] Q. Ren, Y. Dong, and T. Yu, "Numerical modeling of concrete hydraulic fracturing with extended finite element method," Science in China Series E: Technological Sciences, vol. 52, no. 3, pp. 559-565, 2009.

[10] Q. Zeng and J. Yao, "Numerical simulation of fracture network generation in naturally fractured reservoirs," Journal of Natural Gas Science and Engineering, vol. 30, pp. 430-443, 2016.

[11] O. Kresse, X. Weng, and M. Mack, "Numerical modeling of 3D hydraulic fractures interaction in complex naturally fractured formations," Rock Mechanics and Rock Engineering, vol. 51, no. 12, pp. 3863-3881, 2018.

[12] F. Zhang, E. Dontsov, and M. Mack, "Fully coupled simulation of a hydraulic fracture interacting with natural fractures with a hybrid discrete-continuum method," International Journal for Numerical and Analytical Methods in Geomechanics, vol. 41, no. 13, pp. 1430-1452, 2017.

[13] L. Huang, J. Liu, F. Zhang, H. Fu, H. Zhu, and B. Damjanac, "3D lattice modeling of hydraulic fracture initiation and nearwellbore propagation for different perforation models," Journal of Petroleum Science and Engineering, vol. 191, p. $107169,2020$.

[14] X. Guo, H. Song, K. Wu, and J. Killough, "Pressure characteristics and performance of multi-stage fractured horizontal well in shale gas reservoirs with coupled flow and geomechanics," Journal of Petroleum Science and Engineering, vol. 163, pp. 1-15, 2018.

[15] F. Zhang, B. Damjanac, and S. Maxwell, "Investigating hydraulic fracturing complexity in naturally fractured rock masses using fully coupled multiscale numerical modeling," Rock Mechanics and Rock Engineering, vol. 52, no. 12, pp. 5137-5160, 2019.

[16] T. Belytschko and T. Black, "Elastic crack growth in finite elements with minimal remeshing," International Journal for Numerical Methods in Engineering, vol. 45, no. 5, pp. 601-620, 1999.

[17] A. Dahi-Taleghani and J. E. Olson, "Numerical modeling of multistranded-hydraulic-fracture propagation: accounting for the interaction between induced and natural fractures," SPE Journal, vol. 16, no. 03, pp. 575-581, 2011.

[18] A. Dahi Taleghani and J. E. Olson, "How natural fractures could affect hydraulic-fracture geometry," SPE Journal, vol. 19, no. 01, pp. 161-171, 2013.

[19] D. Xu, Z. Liu, X. Liu, Q. Zeng, and Z. Zhuang, "Modeling of dynamic crack branching by enhanced extended finite element method," Computational Mechanics, vol. 54, no. 2, pp. 489-502, 2014.

[20] X. Wang, F. Shi, C. Liu, D. Lu, H. Liu, and H. Wu, "Extended finite element simulation of fracture network propagation in formation containing frictional and cemented natural fractures," Journal of Natural Gas Science and Engineering, vol. 50, pp. 309-324, 2018.

[21] D. Xu, Z. Liu, Z. Zhuang, Q. Zeng, and T. Wang, "Study on interaction between induced and natural fractures by extended finite element method," SCIENCE CHINA Physics, Mechanics \& Astronomy, vol. 60, no. 2, Article ID 024611, 2017.

[22] Z. Zhuang and B.-B. Cheng, "Development of X-FEM methodology and study on mixed-mode crack propagation," Acta Mechanica Sinica, vol. 27, no. 3, pp. 406-415, 2011. 